\title{
BMJ Open Prophylactic epinephrine for the prevention of transbronchial lung biopsy-related bleeding in lung transplant recipients (PROPHET) study: a protocol for a multicentre randomised, double-blind, placebo-controlled trial
}

Or Kalchiem-Dekel, ${ }^{1}$ Aldo lacono, ${ }^{2}$ Edward M Pickering, ${ }^{1}$ Ashutosh Sachdeva, ${ }^{1}$ Nirav G Shah, ${ }^{1}$ Mark Sperry, ${ }^{1}$ Bich-Chieu Tran, ${ }^{1}$ Robert M Reed ${ }^{\oplus 1}$

To cite: Kalchiem-Dekel 0 , lacono A, Pickering EM, et al. Prophylactic epinephrine for the prevention of transbronchial lung biopsy-related bleeding in lung transplant recipients (PROPHET) study: a protocol for a multicentre randomised, double-blind, placebocontrolled trial. BMJ Open 2019;9:e024521. doi:10.1136/ bmjopen-2018-024521

- Prepublication history for this paper is available online. To view these files, please visit the journal online (http://dx.doi. org/10.1136/bmjopen-2018024521).

Received 31 May 2018 Revised 20 February 2019 Accepted 21 February 2019

Check for updates

(C) Author(s) (or their employer(s)) 2019. Re-use permitted under CC BY-NC. No commercial re-use. See rights and permissions. Published by BMJ.

For numbered affiliations see end of article.

Correspondence to

Dr Robert M Reed;

rreed@som.umaryland.edu

\section{ABSTRACT}

Introduction Transbronchial lung biopsy (TBLB) is frequently performed in single-lung and double-lung transplant recipients for evaluation of clinical and radiological findings as well as routine surveillance for acute cellular rejection. While rates of clinically significant TBLB-related haemorrhage are $<1 \%$ for all comers, the incidence in lung transplant recipients is reported to be higher, presumably due to persistent allograft inflammation and alterations in allograft blood flow. While routinely performed by some bronchoscopists, the efficacy and safety profile of prophylactic administration of topical intrabronchial diluted epinephrine for the prevention of TBLB-related haemorrhage has not been explored in a prospective manner.

Methods and analysis In this randomised, double-blind, placebo-controlled multicentre trial (PROPHET Study), single-lung and double-lung transplant adult recipients from participating institutions who are scheduled for bronchoscopy with TBLB for clinical indications will be identified. Potential participants who meet inclusion and exclusion criteria and sign an informed consent will be randomised to receive either diluted epinephrine or placebo prior to performance of TBLB. The degree of TBLBrelated haemorrhage will be graded by the performing bronchoscopist as well as independent observers. The primary analysis will compare the rates of severe and very severe bleeding in participants treated with epinephrine or placebo. The study will also evaluate the safety profile of prophylactic topical epinephrine including the occurrence of serious cardiovascular and haemodynamic adverse events. Additional secondary outcomes to be explored include rates of non-severe TBLB-related haemorrhage, overall yield of the bronchoscopic procedure and nonserious cardiovascular and haemodynamic adverse effects. Ethics and dissemination The study procedures were reviewed and approved by institutional review boards in participating institutions. This study is being externally monitored, and a data and safety monitoring committee has been assembled to monitor patient safety and to evaluate the efficacy of the intervention. The results of this
Strengths and limitations of this study

- Randomised, double-blinded, placebo-controlled multicentre design to ensure unbiased treatment effect measure.

- Comprehensive data are collected, including video-recording of bronchoscopic procedures, to allow independent evaluation by observers blinded to the individual participant data and study arm allocation.

- Study outcome to be evaluated comprehensively via several grading scales by both procedure performer and independent observers.

- The target population of the study, while enriching the outcome of interest, may limit generalisability of the findings to other populations.

- Data informing optimal dose of topical epinephrine and volume of instillation are lacking. Dose and volume used in this study are based on local expertise and may not necessarily represent optimal values.

study will be published in peer-reviewed scientific journals and presented at relevant academic conferences.

Trial registration number NCT03126968; Pre-results.

\section{INTRODUCTION}

Flexible bronchoscopy with transbronchial lung biopsy (TBLB) has become a central diagnostic modality for assessment of lung allograft function and specifically acute cellular rejection (ACR) following lung transplantation. Most lung transplant programmes now perform TBLB for allograft function surveillance, new onset symptoms, $\geq 10 \%$ decrease in forced expiratory volume over $1 \mathrm{~s}$, assessment of new findings on chest imaging or as a follow-up for acute rejection or cytomegalovirus pneumonitis. ${ }^{1}$ For ACR surveillance in lung and lung-heart 
transplant recipients, most transplant centres will obtain at least 5 and up to 10 biopsies per procedure. ${ }^{2}{ }^{3}$ The frequency and period of time over which surveillance biopsies are obtained is debatable and based on local expertise within transplant institutions.

TBLB is generally safe, although severe and life-threatening procedure-related complications are occasionally encountered. Most common among them are pneumothorax and haemorrhage. Large population-based studies have established the overall rate of TBLB-related haemorrhage, defined arbitrarily as recovery of $\geq 50 \mathrm{~mL}$ of bloody fluid, at $0.58 \%-0.73 \% .{ }^{45}$ Death related to airway bleeding in association with bronchoscopic procedures is exceedingly rare. ${ }^{56}$ Evidence suggests a higher propensity for TBLB-related bleeding in the lung and heart-lung transplant population. In a population of lung-heart transplant patients, Scott $e$ t $a l^{2}$ recorded a $12.6 \%$ rate of $>100 \mathrm{~mL}$ procedure-related bleeding. In another prospective study, Diette et $a l^{7}$ recorded higher rates of bronchoscopy-related haemorrhage, postprocedure haemoptysis and early termination of a procedure due to bleeding in lung transplant patients when compared with non-transplant controls. Possible reasons posited to explain the greater propensity to bleed among lung transplant recipients include an already inflamed lung tissue from infection, ACR or bronchiolitis obliterans, as well as an increase in blood flow to the transplanted lung, particularly in recipients of single lung transplants. ${ }^{7}$

Quantification of TBLB-related bleeding is difficult, subjective and prone to bias. Many investigators use grading systems that rely on the amount of mixed blood and bronchial wash fluid in the vacuum suction system at the end of the procedure. ${ }^{5-10}$ This method may be prone to measurement errors in estimation of the actual extent of blood loss during the procedure due to dilution of blood with other airway secretions as well as fluids administered via the bronchoscope channel. Pilarczyk $e t$ $a l^{11}$ suggested a grading system that classifies endobronchial haemorrhage based on the most proximal bronchus in the bronchial tree obstructed by blood. This system is imperfect in the sense that severe haemorrhage can be controlled locally before blood spillage into the more proximal bronchial tree, thus resulting in underestimation of the degree of bleeding. The system suggested by Herth et al grades airway bleeding according to the action implemented by the operator to achieve haemostasis. ${ }^{12} 13$ This system may be biased by the fact that different operators may have different thresholds for implementation of various interventions to control haemorrhage.

Airway bleeding following TBLB can frequently be managed in the bronchoscopy suite. Initial control of haemorrhage can be achieved by lodging the bronchoscope or a balloon catheter in the airway to achieve a tamponade effect. Other measures include placing the patient in the lateral decubitus position with the bleeding lung in the dependent position and topical application of iced saline, ${ }^{14}$ vasopressors, such as cocaine or diluted epinephrine, ${ }^{15} 16$ and haemostatic agents, such as tranexamic acid. ${ }^{17} 18$ Dosing of topical epinephrine for treatment of airway bleeding lacks standardisation. Recommendations regarding epinephrine dilution range between 1:10000 and 1:100 000 and recommended volumes of instillation vary between $0.5 \mathrm{~mL}$ and $20 \mathrm{~mL}$ in different publications. ${ }^{16}{ }^{19-21}$ Our local practice for the initial treatment of endobronchial bleeding includes administration of iced saline in aliquots of $2-10 \mathrm{~mL}$ followed by $1-2 \mathrm{~mL}$ aliquots of 1:10000 topical epinephrine $(0.1-0.2 \mathrm{mg})$ to a maximum of $6 \mathrm{~mL}(0.6 \mathrm{mg})$.

Although adopted by some providers as means to reduced TBLB-related haemorrhage, the use of prophylactic instillation of diluted epinephrine into the target bronchus before attempting TBLB is not established in the literature. The main body of evidence regarding the efficacy and safety of prophylactic topical epinephrine for the prevention of haemorrhage is derived from the surgical literature, specifically endoscopic endonasal and sinus surgery, rhinoplasty and surgical management of burn injuries. $^{22-25}$

The potential for endobronchially administered epinephrine to result in adverse haemodynamic effects is well recognised, and dosing recommendations have been made since the 1970s. ${ }^{26}$ Nevertheless, evidence of such adverse effects is scarce. Janjua $e t a l^{27}$ reported a case of coronary vasospasm manifesting as chest pain, ST-segment elevation and ectopy induced by topical administration of $3 \mathrm{~mL}$ of 1:10000 diluted epinephrine during bronchoscopy. In this case, epinephrine was administered into inflamed and friable airways of a lung cancer patient for the treatment of established bleeding, potentially augmenting the area of contact between epinephrine and injured blood vessels. More recently, two cases of ventricular fibrillation possibly induced by bronchoscopic administration of epinephrine for TBLB-related bleeding were reported.$^{28-30}$ In both cases, arrhythmia occurred with bronchoscopic instillation of epinephrine as a therapeutic measure in response to bleeding and via a catheter that was positioned distally in the bronchial tree and in close proximity to a bleeding vessel as well as the thin alveolar-capillary interface, which allows more systemic absorption of epinephrine.

\section{OBJECTIVE}

The primary efficacy objective of this multicentre, randomised, double-blind, placebo-controlled trial is to determine whether instillation of topical epinephrine versus placebo prior to performance of TBLB will decrease the frequency of biopsy-related severe and very severe haemorrhage in lung transplant recipients.

The secondary objectives of the study are to evaluate the effect of the study intervention on: (1) the extent of biopsy related haemorrhage as assessed by the performing bronchoscopist as well as two independent observers; (2) total procedure efficiency in terms of procedure duration, incidence of early termination due to bleeding complication, achievement of prespecified target number of adequate 
tissue samples and number of pathologically adequate tissue samples obtained; (3) incidence of interventions performed to achieve haemostasis; and (4) incidence of escalation of care due to bleeding complications.

The safety objectives of this study are to determine whether instillation of topical epinephrine prior to performance of TBLB is associated with serious haemodynamic or cardiovascular adverse effects as compared with placebo.

\section{Specific objectives and hypotheses}

\section{Aim 1}

To evaluate the hypothesis that prophylactic administration of topical epinephrine reduces TBLB-related haemorrhage in lung transplant recipients, including:

1. Subjective assessments of bleeding performed by individuals blinded to intervention.

2. The frequency of measures taken to control endobronchial haemorrhage once it occurred.

3. Evaluation for differential response between single-lung and double-lung transplant recipients.

4. Identification of clinical factors associated with an increased or decreased risk of procedure-related haemorrhage.

\section{Aim 2}

To evaluate the hypothesis that prophylactic administration of topical epinephrine affects the overall efficiency of bronchoscopy with TBLB performance, including:

1. The duration of the bronchoscopic procedure.

2. The proportion of procedures completed as planned in terms of number of adequate biopsies obtained as assessed by the bronchoscopist.

3. The proportion of procedures resulting in acquisition of adequate biopsy samples that allow proper pathological evaluation and assignment of a pathological diagnosis.

\section{Aim 3}

To explore the hypothesis that instillation of prophylactic topical epinephrine into the target biopsy airway is not associated with an adverse event (AE) profile significantly different from placebo, including:

1. Frequency of clinically significant haemodynamic derangements, including hypertension and tachycardia.

2. Frequency of cardiac AEs, including conduction abnormalities, arrhythmia and myocardial ischaemia.

3. Frequency of other vascular AEs, including stroke, mesenteric ischaemia and critical limb ischaemia.

4. To identify clinical factors associated with an increased or decreased risk of drug-related AEs.

\section{Aim 4}

To assess new tools for the standardised grading of TBLB-related and other bronchoscopic procedures-related haemorrhage:

1. Assessment of a five-scale grading tool intended for use by the performing bronchoscopist, which represents a modification of the grading scale suggested by Herth et al. ${ }^{12}$

2. Assessment of a four-scale grading tool intended for use by observers viewing the procedural video recording, which represents a modification of the grading scale suggested by Pilarczyk et al. ${ }^{11}$

3. Assessment of the degree of agreement between the two above-mentioned grading scales in the definition of severe haemorrhage.

\section{METHODS}

Reporting of this study protocol has been verified in accordance with the Standard Protocol Items for Randomized Trials recommendations.

\section{Eligibility}

Male and female potential participants $\geq 18$ years of age, who are recipients of single-lung or double-lung transplantation and were scheduled for clinically indicated bronchoscopy with TBLB will be eligible for inclusion. All eligible candidates must be willing to sign an informed consent to participate in the study. As delineated in more detail in Box 1, potential participants will be excluded if: (1) pregnant; (2) there is an absolute or relative contraindication to TBLB; or (3) there is an absolute or relative contraindication for application of topical epinephrine.

\section{Recruitment}

Potential trial participants will be screened from the patient cohort being followed and treated by the lung transplantation service at participating study centres. Screening will apply only to eligible participants requiring TBLB, based on standards of care at participating centres. Informed consent will be obtained on the day of study recruitment. Only a well-trained clinical coordinator, the principal investigator (PI) or an associate investigator will obtain consent. Details of the protocol will be discussed with the potential participants at the time of scheduling the bronchoscopy procedure, ensuring $>24$ hours for consideration of the protocol. These individuals will then be approached with the informed consent form. The consent will be obtained using an institutional review board (IRB)-approved and stamped consent form. Whenever possible, the informed consent form will be given to the potential participants in advance.

Lung transplantation recipients frequently undergo multiple bronchoscopic procedures in the post-transplantation period. Hence, it is likely that a subject who is eligible for participation in the study will undergo more than one bronchoscopy with TBLB during the study period. In this instance, a separate informed consent will be obtained for each individual procedure.

\section{Enrolment}

As summarised in box 2, at enrolment each study participant's medical record will be reviewed for demographic data, past medical history and comorbid conditions, current medication regimen, indication for 


\section{Box 1 Exclusion criteria}

1. General:

1.1. Pregnancy.

2. Contraindications for TBLB:

2.1. Platelet count $<50 \mathrm{~K} / \mu \mathrm{L}$.

2.2. INR $>1.5$.

2.3. Known bleeding diathesis.

2.4. Use of prophylactic or therapeutic dose of unfractionated heparin within 6 hours of the procedure.

2.5. Use of prophylactic dose of low molecular weight heparin within 12 hours of the procedure.

2.6. Use of therapeutic dose of low molecular weight heparin within 24 hours of the procedure.

2.7. Use of oral direct thrombin inhibitors or oral factor 10a inhibitors within 48 hours of the procedure.

2.8. Use of clopidogrel, ticlopidine, ticagrelor or prasugrel within 5 days of the procedure.

2.9. Renal failure, defined as estimated glomerular filtration rate $\leq 30 \mathrm{~mL} / \mathrm{min}$.

2.10. Severe pulmonary hypertension as defined by a mean pulmonary artery pressure of $>40 \mathrm{~mm} \mathrm{Hg}$ on right heart catheterisation or an estimated pulmonary artery systolic pressure of $>62 \mathrm{~mm} \mathrm{Hg}$ on transthoracic echocardiography, both performed within 1 year of the index procedure.

2.11. An additional synchronous procedure with possible bleeding (BAL and endobronchial biopsy allowed), unless the additional procedure is to be performed following completion of TBLB.

2.12. Decompensated liver cirrhosis, defined as the presence of clinically significant ascites, clinical evidence of esophageal or gastric varices or history of bleeding from gastric or oesophageal varices.

2.13. Prior history of TBLB-related airway bleeding requiring admission to the hospital or advanced measures to achieve haemostasis, including endotracheal intubation, bronchial blocker application, bronchial artery embolisation or surgical intervention.

3. Contraindications for topical epinephrine:

3.1. Systolic heart failure with an ejection fraction of $<35 \%$ as assessed by echocardiography performed within 1 year prior to the index procedure.

3.2. Myocardial infarction, acute coronary syndrome, percutaneous coronary intervention or coronary artery bypass surgery within 6 months prior to the index procedure.

3.3. Symptoms and/or ECG findings suggestive of ongoing cardiac ischaemia on the day of the index procedure.

3.4. Moderate-grade to severe-grade aortic or mitral cardiac valvulopathy as assessed by echocardiography performed within 1 year prior to the index procedure.

3.5. Inadequately controlled supraventricular arrhythmia, including atrial fibrillation, atrial flutter and atrioventricular node re-entrant tachycardia as revealed by ECG or cardiac monitoring at the day of the index procedure.

3.6. Presence of an internal cardioverter/defibrillator.

3.7. History of second-degree or third-degree heart block or sick sinus syndrome.

3.8. Baseline ECG or cardiac monitoring revealing $\geq 10$ events per minute of atrial or ventricular ectopy as documented prior to or at the time of the procedure.

3.9. History of ventricular arrhythmias requiring pharmacological or electrical cardioversion within 60 days preceding the index procedure.

3.10. Serum potassium of $<3.0 \mathrm{mmol} / \mathrm{L}$ within the week prior to the index procedure.

3.11. Serum glucose level of $\geq 300 \mathrm{mg} / \mathrm{dL}$ within the week prior to the index procedure.

3.12. Any history of critical ischaemia related to peripheral arterial disease.

3.13. Persistent resting heart rate measurement of $\geq 120$ beats per minute prior to or at the time of the index procedure.

3.14. Persistent resting systolic blood pressure measurement of $\geq 180 \mathrm{~mm} \mathrm{Hg}$ prior to or at the time of the index procedure.

3.15. Persistent resting diastolic blood pressure measurement of $\geq 110 \mathrm{~mm} \mathrm{Hg}$ prior to or at the time of the index procedure.

3.16. History of acute closed-angle glaucoma within 1 year of the procedure.

3.17. Diagnosis of pheochromocytoma requiring pharmacological therapy with an alpha adrenoreceptor blocker at the time of the index procedure.

3.18. Diagnosis of thyrotoxicosis requiring pharmacological therapy with an antithyroid agent at the time of the index procedure.

BAL, bronchoalveolar lavage; INR, international normalised ratio; TBLB, transbronchial lung biopsy.BAL, bronchoalveolar lavage; INR, international normalised ratio; TBLB, transbronchial lung biopsy.

lung transplantation, indication for index bronchoscopy with TBLB, possible concurrent procedures, most recent spirometry measurements, most recent report of echocardiogram, laboratory data, 12-lead ECG and the target number of adequate biopsy specimens and target lobes and segments, as designated by the performing bronchoscopist.

\section{Participant monitoring}

On the day of procedure, participants will have intake vital signs obtained. Recent history will be obtained, and physical examination will be performed. Participants will be monitored under telemetry starting with this intake period, during and for approximately 1 hour after conclusion of the procedure or longer if clinically indicated.

\section{Study drug and placebo preparation and packaging}

For this study, we adopted the recommendations made by the British Thoracic Society (BTS) regarding the ratio dilution of epinephrine $1 \mathrm{mg} / \mathrm{mL}$ to $1: 10000 .{ }^{15}$ As for the volume of instillation, we chose a volume of $2 \mathrm{~mL}$ based on our common local practice.

Epinephrine and placebo preparation will be performed on the day of the index procedure by a member of the research team who will remain blinded to selection of the study drug versus placebo. Two syringes containing 
$2 \mathrm{~mL}$ of $1 \mathrm{mg} / \mathrm{mL}$ of epinephrine diluted to $1: 10000$ in sterile room temperature $0.9 \%$ saline along with $8 \mathrm{~mL}$ of ambient air (epinephrine arm) will be drawn. Subsequently, two syringes containing $2 \mathrm{~mL}$ of room temperature sterile $0.9 \%$ saline along with $8 \mathrm{~mL}$ of ambient air (placebo arm) will also be drawn. Saline was chosen as a comparator based on its almost complete similarity to soluble epinephrine in terms of colour and consistency. In addition, our practice is to dilute the epinephrine in $0.9 \%$ saline, making it a suitable control vehicle. The decision to prepare two syringes in each set is to allow for the possibility that biopsies will be attempted from more than one airway. Thereby, if biopsy attempts are performed via two different airways, application of the allocated study drug can be performed twice. Study drug instillation will not be performed with any biopsy attempts beyond the second.

In order not to breach blinding, lot numbers for both epinephrine and saline aliquots will be documented in each study participant's record regardless of study arm allocation

\section{Randomisation}

To eliminate bias, two randomisation and blinding processes will be employed: (1) in preparing and labelling the sets syringes; and (2) in choosing one of the two sets of syringes at the time of the procedure. The two separate randomisation processes will be performed by different members of the research team, each of them blinded to the results of the other randomisation process.

First, the two sets of syringes containing epinephrine and placebo will be labelled 'A' or 'B' based on an electronic coin toss. A record of the coded syringes and their content will be placed in a sealed envelope and later documented in a unique database separate from the general study database. The syringes will be delivered to the procedure room prior to the procedure. Second, the bronchoscopist who is blinded to the drug and placebo preparation process will randomly choose 'A' or 'B' based on an electronic coin toss. A record of the chosen set of syringes will be placed in a sealed envelope and later documented in the unique study database.

By adopting this randomisation system, we ensure blinding of all study-related personnel, including study team member in charge of drug preparation, bronchoscopist, study subject and independent observers. At the completion of the randomisation phase, the study participant will be randomised to the epinephrine or placebo arm for the purpose of the upcoming procedure only.

\section{Intervention}

The study intervention will be performed by designated members of the study team in each participating centre. Study bronchoscopy will be performed by an experienced bronchoscopist, defined for the purpose of this study and in accordance with the American Thoracic Society/European Respiratory Society guidelines, ${ }^{31}$ as $\geq 25$ TBLB procedures performed per year. Additionally, bronchoscopy can be performed by a trainee under direct supervision of an experienced bronchoscopist.

Bronchoscopy will be performed as per local policy and under moderate sedation or general anaesthesia as previously planned by the bronchoscopist based on clinical indications. As per local protocol, periprocedurally and during the procedure, the subject's heart rate, electrocardiography rhythm strip, respiratory rate and oxygen saturation will be monitored continuously. Blood pressure will be repeatedly measured every $3 \mathrm{~min}$. Supplemental oxygen will be provided to maintain an oxygen saturation of $>92 \%$ as measured by pulse oximetry. A defibrillator as well as a resuscitation drugs and equipment must be available at all times in the procedure area.

Premedication will be performed per local protocol and at the discretion of the performing bronchoscopist. All medications given prior to, during and up to 3 hours following the study procedure will be recorded.

For the purpose of independent postprocedure analysis, the entire procedure from insertion of the bronchoscope into the airways until its final withdrawal will be video-recorded on DVD media and individually labelled without disclosing study arm allocation. DVDs will be securely stored with other individual patient study documents for later review by independent observers. Observers will be experienced pulmonologist faculty members with a record of $\geq 500$ bronchoscopy procedures.

Prior to biopsy performance, one of the labelled previously allocated study syringes will be instilled into the target airway by the bronchoscopist. This process may be repeated once with the other syringe, should the performing bronchoscopist choose to perform additional biopsy passes via a different airway. If this is done, the same study drug-be it epinephrine or placebo—will be instilled into both airways. Otherwise stated, there would not be a scenario in which one airway would receive active drug and the other would receive placebo. A maximum of two aliquots will be given.

Biopsies will be performed using bronchoscopic biopsy forceps at the discretion of the bronchoscopist. Use of fluoroscopic guidance will also be left to the discretion of the bronchoscopist.

Data to be recorded during the procedure are summarised in box 2 .

\section{Postprocedure follow-up}

Following termination of the procedure, recovery will be conducted per local policy. Postprocedure, each study subject will be assessed by a study team clinician to evaluate for signs and symptoms of pneumothorax, pulmonary haemorrhage or haemodynamic and cardiac abnormalities on the same day of the procedure. A chest radiograph will be obtained to identify late pneumothorax within 2 hours of the termination of the procedure or later if deemed necessary by clinical judgement. Repeat ECG will be obtained within 2 hours of the termination of the procedure or earlier if symptoms suggestive of cardiac arrhythmia, ischaemia or conduction abnormality appear. 
In the outpatient setting, discharge from the admitting facility will be conducted per local policy. A follow-up telephone call will be performed by one of the study investigators 48 hours following the index procedure. Participants will be requested to report any ill effects of the procedure and will be specifically asked about sore throat, fever, chills, haemoptysis, dyspnoea and chest pain. Symptomatic patients will be managed as deemed appropriate and according to clinical judgement and local policies. In addition, any escalation in the level of care occurring within 48 hours of the index procedure will be recorded. This includes emergency department visits, urgent care provider visits and hospital admissions. The reason leading to escalation in the level of care will also be recorded.

In the inpatient setting, all study-related procedures, including preprocedure work-up, recruitment, enrolment, preprocedure and postprocedure monitoring and postprocedure follow-up will be performed in the admitting unit by the study investigators. Escalation in the level of care in the inpatient setting will include transfer to a higher level of care unit.

Data to be recorded postprocedure are summarised in Box 2. A typical time line for outpatient study screening,

\section{Box 2 Study data acquisition}

\section{Preprocedure data:}

1.1. Demographic data (age, gender, race and ethnicity).

1.2. Transplant-related data (indication, laterality, date of surgery and heart transplantation).

1.3. Comorbidities.

1.4. Current medication list.

1.5. Recent ECG, spirometry, echocardiography and laboratory data.

1.6. Current indication for TBLB.

1.7. Target TBLB sites and target number of visualised adequate specimen.

1.8. Study epinephrine and normal saline lot numbers.

1.9. Study syringe drug labelling (to be placed in a separate, sealed envelope).

1.10. Study subject drug allocation (to be placed in a separate, sealed envelope).

2. Procedure-related data:

2.1. Bronchoscope model.

2.2. Approach (transoral, transnasal, endotracheal tube and tracheostomy tube).

2.3. Biopsy forceps model and measures.

2.4. Timing:

2.4.1. First bronchoscope insertion.

2.4.2. Instillation of first study drug.

2.4.3. Instillation of second study drug (optional).

2.4.4. Final bronchoscope removal.

2.5. Use of fluoroscopy and total fluoroscopy time.

2.6. Number of biopsy forceps passes by segment.

2.7. Number of visualised adequate biopsy samples obtained by segment.

2.8. Performance of BAL (timing, lobe, segment, volume instilled and volume returned).

2.9. Performance of other bronchoscopic procedures.

2.10. Telemetry recordings of blood pressure, heart rate, ECG and oxygen saturation.

2.11. Any supplemental oxygen, mode of delivery and flow rate.

2.12. Any haemodynamic, cardiac or respiratory events not otherwise captured by telemetry.

2.13. All medications provided.

2.14. Bleeding-related and other interventions performed to control procedure-related adverse events.

2.15. Total volume and appearance of fluid in collection containers.

2.16. Reason for termination of procedure; if procedure terminated not as anticipated, reason need to be provided.

2.17. Video recording of the procedure from initial bronchoscope insertion to final bronchoscope removal.

3. Postprocedure data:

3.1. Mortality within 3 hours of study drug instillation.

3.2. Grade of bleeding by performing bronchoscopist (primary efficacy outcome).

3.3. Grade of bleeding by independent observers.

3.4. Findings on postprocedure roentgenogram.

3.5. Other events occurring between termination of procedure and discharge or transfer back to admission unit.

3.6. Escalation in level of care in the time period immediately following termination of the procedure.

3.7. Number of pathologically adequate specimens.

3.8. Histopathological diagnosis.

3.9. Surveillance at 48 hours postprocedure (fever, chills, haemoptysis, dyspnoea and chest pain) and any interventions performed or escalation in level of care.

BAL, bronchoalveolar lavage; TBLB, transbronchial lung biopsy.BAL, bronchoalveolar lavage; TBLB, transbronchial lung biopsy. 
Table 1 Timeline for study outpatient screening, recruitment, enrolment, participation and follow-up

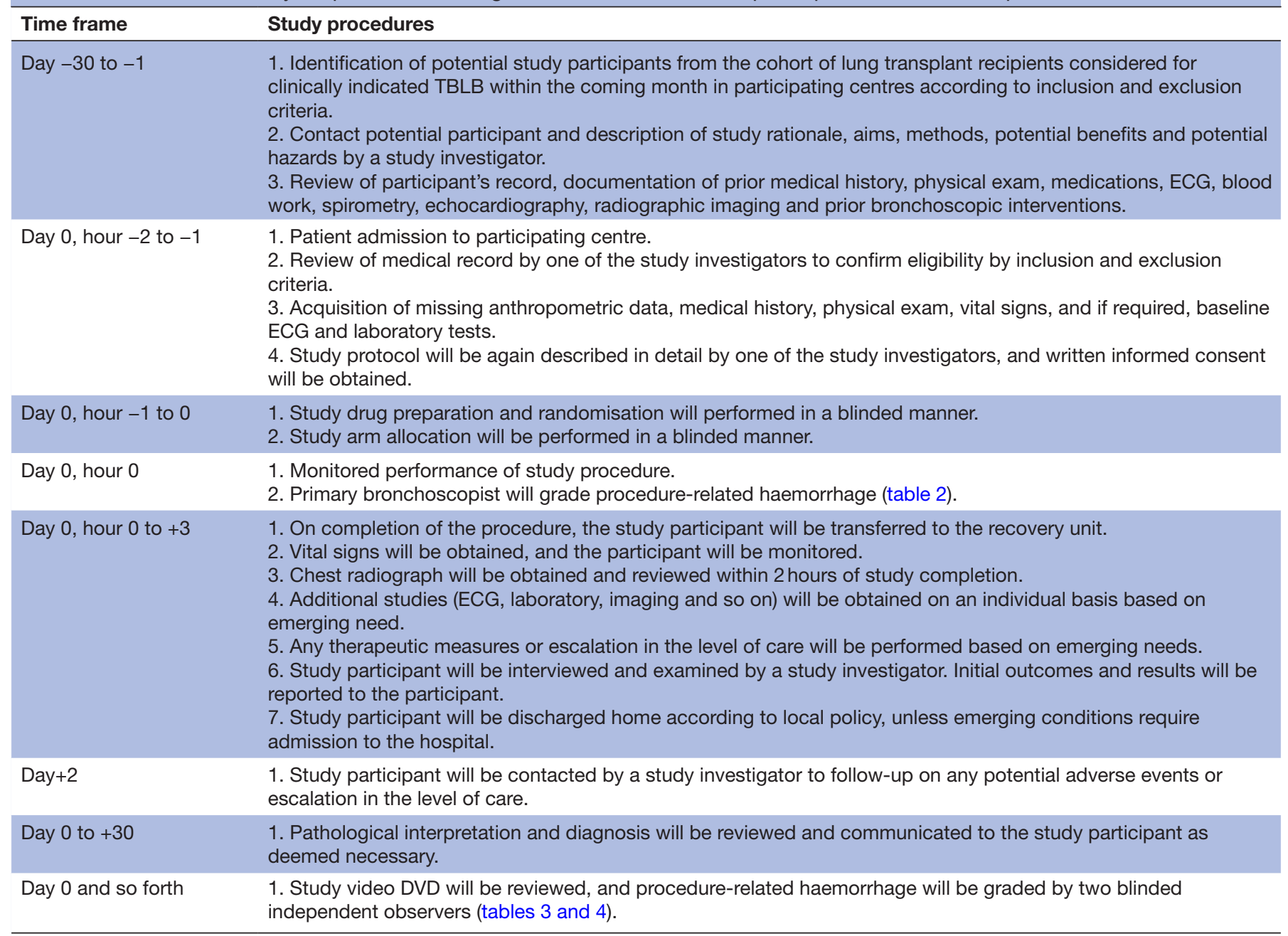

TBLB, transbronchial lung biopsy.

recruitment, enrolment, participation and follow-up is summarised in table 1.

\section{Criteria for withdrawal of participants}

In the event a study subject elects to be withdrawn from the study and requests their data to be withdrawn as well, we will comply.

\section{Study outcomes}

Primary efficacy outcome

The primary efficacy outcome of the PROPHET Study is the incidence of severe or very severe haemorrhage as defined by the performing bronchoscopist. As summarised in table 2, TBLB-related haemorrhage will be graded according to the grading system suggested by Herth $e t a l^{12}$ and modified in accordance with the PROPHET Study scientific question. Pilarczyk $e t \mathrm{al}^{11}$ and modified in accordance with the PROPHET Study scientific question.

\section{Secondary efficacy outcomes}

The secondary efficacy outcomes of the PROPHET study are: (1) occurrence of no, mild or moderate haemorrhage as defined by the bronchoscopist; (2) general assessment of degree of TBLB-related bleeding as indicated by the independent observer using the grading scale suggested by Pilarczyk $e t a l^{11}$ and modified in accordance with the PROPHET Study scientific question (table 3); (3) general assessment of degree of TBLB-related bleeding as indicated by the independent observer using a Likert scale (table 4); (4) number of forceps passes performed per biopsy site; (5) number of visualised adequate tissue samples obtained; (6) number of pathologically adequate tissue samples obtained; (7) achievement of histological diagnosis; (8) early termination of the procedure due to bleeding complication; (9) duration of the procedure from first bronchoscope insertion to final bronchoscope withdrawal; (10) total volume of iced saline used during the procedure; (11) total dose of unblinded topical epinephrine or other haemostatic agent used during the procedure; (12) performance of advanced haemostatic measures, including balloon tamponade, application of endobronchial blocker, bronchial artery embolisation and/or emergency surgery in response to haemorrhagic complcation; (13) use of devices or procedures for securing the airway, including oral airway, nasal airway, 
Table 2 Intervention-based grading system for TBLB-related haemorrhage by performing bronchoscopist*

\begin{tabular}{ll}
\hline Grade & Description \\
\hline No haemorrhage & No haemorrhage. \\
Mild & $\begin{array}{l}\text { Any bleeding originating from the biopsy target airway requiring wedging of the bronchoscope or 'in } \\
\text { and out' motion in order to achieve haemostasis. }\end{array}$ \\
Moderate & $\begin{array}{l}\text { Any bleeding originating from the biopsy target airway requiring in addition to manoeuvring the } \\
\text { bronchoscope, application of iced saline or topical epinephrine, or placing the patient with the } \\
\text { bleeding lung in the dependent position. }\end{array}$ \\
Severe & $\begin{array}{l}\text { Any bleeding originating from the biopsy target airway requiring, in addition to the above-mentioned } \\
\text { manoeuvres, early termination of the procedure. }\end{array}$ \\
Very severe & $\begin{array}{l}\text { Any bleeding originating from the biopsy target airway requiring, in addition to the above-mentioned } \\
\text { manoeuvres, early termination of the procedure, application of balloon tamponade, endobronchial } \\
\text { intubation, application of a bronchial blocker, or use of other invasive measure to achieve } \\
\text { haemostasis, such as bronchial artery embolisation or surgical intervention. }\end{array}$
\end{tabular}

${ }^{*}$ Adopted with modification from Herth et al. ${ }^{12}$

TBLB, transbronchial lung biopsy.

laryngeal mask airway, endotracheal intubation, emergency cricothyroidotomy and emergency tracheostomy; and (14) unplanned admission to the hospital or transfer to a higher level of care within hospital within 48 hours of the procedure.

\section{Safety outcomes}

The safety of topical epinephrine administration and potential instillation-related AEs will be ascertained from clinical monitoring, vital signs and ECG recordings during and immediately following the index procedure as well as from any clinical, laboratory or radiological investigations performed during or following the index procedure. Complications deemed possibly related to topical epinephrine administration comprising the study safety outcomes include: (1) all-cause mortality within 3 hours of the last instillation of the study drug; (2) acute cardiovascular event, including cardiac arrest, new onset chest pain clinically deemed to be of cardiac origin, acute myocardial ischaemia, pulmonary oedema, acute ischaemic stroke, critical limb ischaemia and acute mesenteric ischaemia; all occurring within $30 \mathrm{~min}$ of the

Table 3 Bronchial tree spillage-based system for grading of TBLB-related haemorrhage by independent study observers*

\begin{tabular}{ll}
\hline Grade & Description \\
\hline No haemorrhage & $\begin{array}{l}\text { No haemorrhage. } \\
\text { Minor }\end{array}$ \\
$\begin{array}{l}\text { Endobronchial haemorrhage resulting in } \\
\text { segmental or more distal bronchus blood } \\
\text { spillage }\end{array}$ \\
Intermediate & $\begin{array}{l}\text { Endobronchial haemorrhage resulting in lobar } \\
\text { bronchus blood spillage. }\end{array}$ \\
Major & $\begin{array}{l}\text { Endobronchial haemorrhage resulting in } \\
\text { mainstem bronchus, more proximal blood } \\
\text { spillage or spillage into the uninvolved lung. }\end{array}$ \\
\hline
\end{tabular}

${ }^{*}$ Adopted with modification from Pilarczyk et al. ${ }^{11}$

TBLB, transbronchial lung biopsy. last instillation of the study drug; (3) acute occurrence of closed-angle glaucoma within $30 \mathrm{~min}$ of the last instillation of the study drug; (4) new onset ECG changes suggestive of myocardial ischaemia, including ST-segment changes, defined as $\geq 1 \mathrm{~mm}$ ST-segment elevation or $\geq 3 \mathrm{~mm}$ ST-segment depression in $\geq 2$ precordial or limb ECG leads that correspond together to one of the cardiac walls, new onset ventricular tachycardia, $\geq 10$ beats per minute increase in occurrence of ventricular ectopic beats, new onset supraventricular arrhythmia, new onset bundle branch block or new onset second-degree or third-degree atrioventricular block, all occurring within $30 \mathrm{~min}$ of the last instillation of the study drug; (5) significant change in heart rate, defined as heart rate of $>120 \mathrm{bpm}$ and/or increase by $\geq 30 \mathrm{bpm}$ above the baseline or heart rate $<60 \mathrm{bpm}$ and/or $\geq 30 \mathrm{bpm}$ below the baseline and as recorded within $30 \mathrm{~min}$ of the last instillation of the study drug; and (6) significant change in systolic blood pressure, defined as increase in systolic blood pressure $>180 \mathrm{~mm} \mathrm{Hg}$ and/or $\geq 40 \mathrm{~mm}$ $\mathrm{Hg}$ above the baseline, increase in diastolic blood pressure $>110 \mathrm{~mm} \mathrm{Hg}$ and/or $\geq 20 \mathrm{~mm} \mathrm{Hg}$ above the baseline or fall in systolic blood pressure $<90 \mathrm{~mm} \mathrm{Hg}$ and/ or $\geq 20 \mathrm{~mm} \mathrm{Hg}$ below the baseline, all recorded within $30 \mathrm{~min}$ of the last instillation of the study drug.

Table 4 Likert scale for grading of TBLB-related haemorrhage by independent study observers

\begin{tabular}{ll}
\hline Grade & Description \\
\hline 1 & Much less than usual bleeding for TBLB. \\
2 & Less than usual bleeding for TBLB. \\
3 & Usual bleeding for TBLB. \\
4 & More than usual bleeding for TBLB. \\
5 & Much more than usual bleeding for TBLB. \\
\hline
\end{tabular}

TBLB, transbronchial lung biopsy. 


\section{Potential risks and discomforts}

The decision to perform bronchoscopy with TBLB as well as the type of sedation administered will be based on clinical indications as a part of each study participant's standard of care and not as a part of the research. Potential risks and discomforts of the bronchoscopy and TBLB themselves are no more than what the participant would undergo as a part of their post-transplantation bronchoscopy.

Bronchoscopic procedures performed in lung transplant patients are usually performed under conscious sedation. Potential risks and discomforts of conscious sedation for this study's participants are no more than what the participant would undergo for their post-transplantation bronchoscopy. For procedures performed under general anaesthesia, potential risks and discomforts of general anaesthesia for this study's participants are no more than what the participant would undergo for their post-transplantation bronchoscopy under general anaesthesia.

The potential risks of epinephrine are well described in cases of sufficient systemic absorption of the drug. Most serious complications are arrhythmias and haemodynamic compromise and tend to occur in a dose-dependent manner. ${ }^{32}$ Most complications described in the literature occurred are in the setting of existing bleeding from procedural maneuvers ${ }^{27-30} 3334$ or in the presence of augmented interface between topical epinephrine and inflamed tissue, such as burn injury. ${ }^{25}{ }^{35}$ Direct communication between epinephrine and blood vessels or inflamed tissues allow more direct absorption of epinephrine leading to higher concentration of epinephrine in the blood stream and therefore systemic complications. There are no reported data describing the incidence rate of complications arising from epinephrine in the setting of prophylactic topical use in the airway, but it is expected to have a lower rate of systemic absorption.

Another research-related risk to participants include loss of confidentiality. Loss of confidentiality is unlikely to occur as all data will be accessed and stored securely only by members of the research team. All patient data will be incorporated into a central database. Patients will be assigned with a unique identification number. The database will be maintained behind firewall in a password-protected folder. The database file will also be password protected. Any limited physical data will be kept in a locked cabinet with only keys available to research team members. Confidential data will not be shared.

This study in not under a master agreement that includes a provision requiring the sponsor to provide compensation to participants for research-related injury.

\section{Data management and confidentiality}

Study-related data will be captured by study team members who are certified in good clinical practice and underwent specific training for the purpose of the current protocol. All study-related data will be anonymised and assigned a unique seven-character study identification number. In each participating centre, individual study participant data will be documented in a designated case report form (CRF). Any information identified as Protected Health Information (PHI) will be kept separate from the participants' CRF in a secure environment at the participating centre accessible only to study staff allowed access to PHI. Each study participant CRF will also include the DVD media used for recording the bronchoscopy procedure. This DVD will only be labelled with the participant's unique identification number and date of recording.

The PROPHET Trial will capture data into one of two computerised databases: (1) all study-related data except study arm allocation and (2) study drug assignment database. The first database will be accessible to investigators blinded to study arm assignment whereas the second database will be accessible only to investigators involved in study drug marking and will remain disconnected from the procedure and outcomes data until unblinding.

For the purpose of maintaining blinding during interim analyses (also see Data analysis), study investigators who are otherwise blinded to the individual participant and procedural data will be unblinded to individual participant study arm allocation. The two study arms will be randomly assigned as 'A' and 'B', and the unblinded investigators will compose a new study drug assignment database where participants will be grouped according to their study arm into either 'A' or 'B'. This will allow analysis of data for safety and efficacy outcomes without breach of blinding for the rest of the study team. Should a signal for possible harm will be observed, the decision to completely unblind study arm assignment will be left to the discretion of the study's data and safety monitor.

All computerised databases will be located at University of Maryland School of Medicine on a local server, behind a network firewall, and accessible only under the following conditions: (1) a workstation physically present in University of Maryland School of Medicine and physically connected to the network; (2) granted system access to database server; (3) granted password to the database folder; and (4) granted password to the database items.

\section{Data analysis}

Formal power calculations are not possible as studies of this kind have not been previously performed and an aim of the study is to develop new bleeding assessment tools. As such, the first 40 procedures will provide data to inform feasibility, safety and more refined power calculations. Numbers are based primarily on feasibility.

Bleeding risk in lung transplantation patients seems to be higher than that of the general population, although one must bear in mind the following caveats: (A) most of the currently published literature is retrospective and observational in nature; (B) lung transplant recipients studies mostly involve small patient populations; and (C) in most studies to date, the definition of clinically significant bleeding was based on the amount of bloody fluid collected in the suction container during the procedure. This quantification method is prone to much bias since 
this fluid is a mixture of blood, airway secretions and lavage fluid, and thus may overestimate the true volume of blood loss. Also, the volume of blood loss considered clinically relevant varies across published studies and affects to a great extent on the reported rate of clinically significant bleeding.

Therefore, the limitation of study participants to transplant recipients is anticipated to enrich the outcome of interest (TBLB-related severe and very severe haemorrhage), and development of a continuous or ordinal assessment measure is expected to improve power compared with a dichotomous outcome measure.

Beyond the main analyses described above, additional data captured during an individual procedure as well as information on challenges encountered in delivery of the intervention will be systematically gathered and noted in designated areas of the CRF to enable further stratified analyses and inform future implementation. These data will also be incorporated it into future iterations of the protocol for multicentre application.

Based on local numbers of lung transplant recipients managed in currently participating centres, we anticipate access to adequate number of participants and procedures in order to complete recruitment.

\section{Statistical analysis}

For the pilot portion of the study, our primary outcome will be the difference in ordinal assessment of bleeding between drug and placebo arms as assessed by the performer of the index procedure (table 2). Initial descriptive summary of the data will be performed. Data will be expressed as means with $\mathrm{SD}$, medians with $\mathrm{IQR}$, percentages and proportions as appropriate. Continuous outcome variables will be visually examined for normality and outliers using histograms and scatter plots. The normal distribution will be formally assessed with the Shapiro-Wilk test. The relationships between continuous variables will be explored by visual inspection. These measures will be compared with continuous variables with the Student's t-test or Mann-Whitney U tests if the data are not normally distributed. Significance level will be set at $\mathrm{p}<0.05$. Differential effects between single and bilateral lung transplants will be examined in both stratified analyses as well as through consideration of an interaction factor in ordered logistic regression equations.

\section{Sample size estimates and power calculations}

The initial 40 participant sample size is based on feasibility and is intended to provide pilot data to inform the full sample size and statistical plan for the larger, more definitive study to follow.

\section{Data and safety monitoring}

A central data and safety monitor or committee will consider all serious adverse events and evaluate the statistical analysis plan and associated termination rules. The monitor or committee will include expertise in clinical trial conductance, statistics, pulmonary care and ethics.
All AEs occurring during the study, including those observed by or reported to the research team, will be recorded. Serious unanticipated problems, defined as complications thought to be likely related to the study drug administration and require some intervention, will be reported to the central monitor and the local IRB as soon as possible but no more than five business days after the PI first learns of the event. Likewise, any serious protocol deviations will be reported to the central committee and the local IRB as soon as possible but no more than seven business days after the PI first learns of the event. Deaths will be reported to the central committee and the local IRB within seven business days after the PI first learns of the event.

Non-serious unanticipated problems will be reported to the central committee and the local IRB as soon as possible but no more than 14 business days after the PI first learns of the event. Non-serious protocol deviations will only be reported to the IRB within 14 business days after the PI first learns of the deviation, if they represent a departure from local institutional policies for the conduct of human subject research, adversely affect the healthcare of study participants or compromise the interpretation or integrity of the research. Non-serious protocol deviations that result from normal subject scheduling variations or technical issues associated with sampling that do not impact the health of the subject or interpretation of the study data will not be reported.

AEs that are clearly not related to the study procedures, such as those that occur prior to initiation of study-related procedures, will not be reported to the central committee or the local IRB. Likewise, AEs that are expected and thought to be related to the natural history of lung transplantation and its comorbidities will not be reported to the central committee or the local IRB. Expected AEs that do not require intervention will not be reported to the central committee or the local IRB. All other AEs will be reported in aggregate at the time of continuing review.

Emergency situations may arise in which it is necessary to unmask treating physicians, emergency department personnel, clinic personnel or the study subject to the assigned treatment. Situations that require unmasking are expected to be rare. Since epinephrine has a short half-life, it will probably be already eliminated from the study participant's circulation by the time they are seen by a provider for possible study drug-related side effect. It is possible, however, that emergency personnel or treating physicians might feel it is necessary to know the medication that participant was receiving to decide on a rational course of treatment or to ensure that other medications are not given that might adversely interact with epinephrine. In such cases, the treating medical personnel will call the PI for study unmasking. In that event, an unmasking report for the event will be completed.

There will be interim analysis of data for safety and all adverse events. These analyses will be done by the central data safety and monitoring committee officer after a pilot period of 40 procedures or 10 major bleeding events, 
whichever occurs first. Thereafter, safety analysis will be performed and reviewed at a minimum of every 12 months. Interim results will be accessible to members of the central committee and the PI, who will reach a mutual decision regarding continuation or termination of the study.

\section{Study timeline}

This version of the study protocol is current as of 25 November 25 2018. Approval for study initiation in University of Maryland Medical Center, Baltimore, Maryland, was obtained by the University of Maryland, Baltimore Professional Schools IRB on 2 June 2017. Recruitment began in USA on 12 June 2017, and participant recruitment is ongoing in University of Maryland Medical Center. On 25 January 2018, University of Maryland Midtown Campus, Baltimore, Maryland, was added as a second recruitment centre. IRB overview for Midtown Campus is provided by University of Maryland, Baltimore Professional Schools IRB per Authorization Agreement signed on 11 March 2015. We anticipate recruitment and enrolment of the first 40 participants and interim analysis by December of 2018. The trial is currently conducted in USA only. A complete list of participating institutions can be found under the study protocol on ClinicalTrials.gov.

\section{Protocol limitations}

Several limitations inherent to this protocol should be noted. The study population eligible for participation in the proposed study is restricted to lung transplant recipients. Given the difference in TBLB-related bleeding propensity among lung transplant recipients when compared with general non-transplanted populations, ${ }^{2,7}$ one should be cautious when attempting to generalise the results of this study to other patient populations undergoing bronchoscopic TBLB. In addition, patient populations with a higher propensity to bleed, such as those with advanced renal failure or liver cirrhosis, are excluded from this protocol. In a similar vein, patient populations with a higher propensity for cardiovascular adverse effect from epinephrine, such as those with recent coronary event, high-grade left-sided valvulopathy or recent cardioversion, are similarly excluded from this study. Therefore, the results of this study may not be generalisable to these patient populations. The dose, volume of instillation and mode of instillation of the study drug in our protocol were based on local practice and expertise. This is due to lack of evidence-based data to support one dose of epinephrine versus another for pulmonary bleeding prophylaxis or for control of established lung haemorrhage. Our choice of epinephrine dose and volume of instillation, however, is supported by the 2013 BTS recommendations for control of TBLB-related haemorrhage. ${ }^{15}$ Currently, there is no standardised scale for grading of the severity of TBLB-related endobronchial haemorrhage. Previously published grading systems were based on estimated volume of bloody fluid in suction collection systems with different volume cut-offs to define severe heamorrhage, proximity of blood spillage into the tracheobronchial tree and operator response and management of bleeding complication. All of the above-mentioned systems are prone to significant bias, due to measurement inaccuracy or operator-dependent factors and preferences. As described in more detail above, in this protocol, we have defined three new grading systems, two of which are based on previous systems ${ }^{11-13}$ (tables 2-4). Grading will be performed by the bronchoscopy operator as well as independent and blinded reviewers of the procedural video recording. Bias is inherent to our grading system as well due to the difference in operator response to bleeding among different bronchoscopists as well as the limitation of grading bleeding by watching a procedural video without knowledge of the happenings outside the bronchoscope view. However, we maintain that the use of several different approaches to grading provide support to the validity of our results.

\section{Ethics and dissemination}

The clinical trial was registered with ClinicalTrials.gov and is accessible to the public since 17 April 2017. Any modifications to the study protocol or consent materials will be submitted for approval by all regulatory authorities before implementation. The trial is being externally monitored, and a central data safety and monitoring committee has been assembled to monitor participant safety and to evaluate the efficacy of the intervention. Results of this study will be disseminated by publication in a peer-reviewed scientific journal and presented at relevant academic conferences. All individuals considered for future authorship of potential publications stemming from this study will have to satisfy all Criteria of Authorship as proposed by the International Committee of Medical Journal Editors. ${ }^{36}$

\section{Patient and public involvement}

Patient stakeholders were not formally engaged in the design of this study.

\section{Author affiliations}

${ }^{1}$ Department of Pulmonary and Critical Care Medicine, University of Maryland School of Medicine, Baltimore, Maryland, USA

${ }^{2}$ Departments of Medicine and Surgery, R Adams Cowley Shock Trauma Center, University of Maryland School of Medicine, Baltimore, Maryland, USA

Contributors All authors listed on this manuscript have reviewed and offered revision suggestions to the manuscript that was drafted by the first and senior authors. All authors listed have also participated in participant enrolment for the initial study period that has informed further refinements of the protocol, and all authors have seen and approved the final manuscript submitted. Study conception and design: OK-D, B-CT, MS and RMR. Acquisition of data: OK-D, B-CT, AS, Al and RMR. Future analysis of data: OK-D, NGS, EMP, AS and RMR. Preparation of manuscript: all authors.

Funding The authors have not declared a specific grant for this research from any funding agency in the public, commercial or not-for-profit sectors.

Competing interests None declared.

Patient consent for publication Not required.

Provenance and peer review Not commissioned; externally peer reviewed. 
Open access This is an open access article distributed in accordance with the Creative Commons Attribution Non Commercial (CC BY-NC 4.0) license, which permits others to distribute, remix, adapt, build upon this work non-commercially, and license their derivative works on different terms, provided the original work is properly cited, appropriate credit is given, any changes made indicated, and the use is non-commercial. See: http://creativecommons.org/licenses/by-nc/4.0/.

\section{REFERENCES}

1. Aboyoun CL, Tamm M, Chhajed PN, et al. Diagnostic value of followup transbronchial lung biopsy after lung rejection. Am J Respir Crit Care Med 2001;164:460-3.

2. Scott JP, Fradet G, Smyth RL, et al. Prospective study of transbronchial biopsies in the management of heart-lung and single lung transplant patients. J Heart Lung Transplant 1991;10:626-7.

3. Kukafka DS, O'Brien GM, Furukawa S, et al. Surveillance bronchoscopy in lung transplant recipients. Chest 1997;111:377-81.

4. Tukey MH, Wiener RS. Population-based estimates of transbronchial lung biopsy utilization and complications. Respir Med 2012;106:1559-65.

5. Asano F, Aoe M, Ohsaki Y, et al. Deaths and complications associated with respiratory endoscopy: a survey by the Japan society for respiratory endoscopy in 2010. Respirology 2012;17:478-85.

6. Flick MR, Wasson K, Dunn LJ, et al. Fatal pulmonary hemorrhage after transbronchial lung biopsy through the fiberoptic bronchoscope. Am Rev Respir Dis 1975;111:853-6.

7. Diette GB, Wiener CM, White P. The higher risk of bleeding in lung transplant recipients from bronchoscopy is independent of traditional bleeding risks: results of a prospective cohort study. Chest 1999;115:397-402.

8. Ibrahim AS, Allangawi MH, Sattar HA, et al. Indications, diagnostic yields and complications of transbronchial biopsy over 5 years in the State of Qatar. Saudi Med J 2005;26:641-5.

9. Hernández Blasco L, Sánchez Hernández IM, Villena Garrido V, et al. Safety of the transbronchial biopsy in outpatients. Chest 1991;99:562-5

10. Chhajed PN, Aboyoun C, Malouf MA, et al. Risk factors and management of bleeding associated with transbronchial lung biopsy in lung transplant recipients. J Heart Lung Transplant 2003;22:195-7.

11. Pilarczyk K, Carstens H, Heckmann J, et al. Safety and efficiency of percutaneous dilatational tracheostomy with direct bronchoscopic guidance for thoracic transplant recipients. Respir Care 2016:61:235-42.

12. Herth FJ, Becker HD, Ernst A. Aspirin does not increase bleeding complications after transbronchial biopsy. Chest 2002;122:1461-4

13. Ernst $A$, Eberhardt $R$, Wahidi $M$, et al. Effect of routine clopidogrel use on bleeding complications after transbronchial biopsy in humans. Chest 2006;129:734-7.

14. Sahebjami H. Letter: Iced saline lavage during bronchoscopy. Chest 1976;69:131-2

15. Du Rand IA, Blaikley J, Booton R, et al. British thoracic society guideline for diagnostic flexible bronchoscopy in adults: accredited by NICE. Thorax 2013;68:i1-44.
16. Lee P, Mehta AC, Mathur PN. Management of complications from diagnostic and interventional bronchoscopy. Respirology 2009;14:940-53.

17. Solomonov A, Fruchter O, Zuckerman T, et al. Pulmonary hemorrhage: a novel mode of therapy. Respir Med 2009;103:1196-200.

18. Márquez-Martín E, Vergara DG, Martín-Juan J, et al. Endobronchial administration of tranexamic acid for controlling pulmonary bleeding: a pilot study. J Bronchology Interv Pulmonol 2010;17:122-5.

19. Ernst A. Introduction to bronchoscopy. 1st edn: Cambridge University Press, 2009.

20. Shah P. Atlas of Flexible Bronchoscopy. 1st edn: CRC Press, 2011.

21. Prakash UBS, Matthay RA. Bronchoscopy. . 1st edn: Raven Pr, 1994:1. 340.

22. Higgins TS, Hwang PH, Kingdom TT, et al. Systematic review of topical vasoconstrictors in endoscopic sinus surgery. Laryngoscope 2011;121:422-32.

23. Valdes CJ, Bogado M, Rammal A, et al. Topical cocaine vs adrenaline in endoscopic sinus surgery: a blinded randomized controlled study. Int Forum Allergy Rhinol 2014;4:646-50.

24. Fernández-Cossío S, Rodríguez-Dintén MJ, Gude F, et al. Topical vasoconstrictors in cosmetic rhinoplasty: comparative evaluation of cocaine versus epinephrine solutions. Aesthetic Plast Surg 2016;40:637-44.

25. Missavage AE, Bush RL, Kien ND, et al. The effect of clysed and topical epinephrine on intraoperative catecholamine levels. J Trauma 1998;45:1074-8.

26. Mall W, Abel $\mathrm{H}$. Topical application of epinephrine during bronchoscopy in barbiturate-halothane-anaesthesia and its influence on cardiac action. Bronchopneumologie 1978;28:311-6.

27. Janjua M, Badshah A, Allen SA. Images in cardiology. Epinephrineinduced ST elevation: a case of endobronchial topical epinephrineinduced coronary vasospasm. Heart 2009;95:656.

28. Steinfort DP, Herth FJ, Eberhardt R, et al. Potentially fatal arrhythmia complicating endobronchial epinephrine for control of iatrogenic bleeding. Am J Respir Crit Care Med 2012;185:1028-30.

29. Khoo KL, Lee P, Mehta AC. Endobronchial epinephrine: confusion is in the air. Am J Respir Crit Care Med 2013;187:1137-8.

30. Steinfort DP, Herth FJ, Eberhardt R, et al. Reply: endobronchial epinephrine: confusion is in the air. Am J Respir Crit Care Med 2013;187:1138.

31. Bolliger CT, Mathur PN, Beamis JF, et al. ERS/ATS statement on interventional pulmonology. European Respiratory Society/American Thoracic Society. Eur Respir J 2002;19:356-73.

32. Roberts JR, Greenberg MI, Knaub MA, et al. Blood levels following intravenous and endotracheal epinephrine administration. JACEP 1979;8:53-6.

33. Korkmaz H, Yao WC, Korkmaz M, et al. Safety and efficacy of concentrated topical epinephrine use in endoscopic endonasal surgery. Int Forum Allergy Rhinol 2015:5:1118-23.

34. Orlandi RR, Warrier S, Sato S, et al. Concentrated topical epinephrine is safe in endoscopic sinus surgery. Am J Rhinol Allergy 2010;24:140-2.

35. Papp AA, Uusaro AV, Ruokonen ET. The effects of topical epinephrine on haemodynamics and markers of tissue perfusion in burned and non-burned patients requiring skin grafting. Burns 2009;35:832-9.

36. Medical Journals. Recommendations for the conduct, reporting, editing, and publication of scholarly work in medical journals. 2017. 